\title{
Human-Robot Collaboration: Task sharing through Virtual Reality
}

\author{
$1^{\text {st }}$ Beibei Shu \\ Department of Industrial Engineering \\ UiT The Arctic University of Norway \\ Narvik, Norway \\ beibei.shu@uit.no
}

\author{
$2^{\text {nd }}$ Gabor Sziebig \\ Department of Industrial Engineering \\ UiT The Arctic University of Norway \\ Narvik, Norway \\ gabor.sziebig@uit.no
}

\author{
$3^{\text {rd }}$ Sakari Pieskä \\ Centria University of Applied Sciences \\ Ylivieska, Finland \\ Sakari.Pieska@centria.fi
}

\begin{abstract}
Collaborative Robots provide many possibilities, when it comes to Human-Robot Collaboration. Until now, these approaches are usually custom made, sensor-integrated solutions, where the robot's safety controller ensures the safety of the human worker. These solutions are according to today's rules and standards. We propose to extend these solutions with including Virtual Realty as a sensor and to provide comfort features to the operator. In order to create cooperation between human and industrial robot in our experiments, we propose to have a simple nut screwing operation as an example, where the industrial robot does the hard part. With sharing the task in such manner, we will ensure that the robot is doing the hard and monotonous work, while the worker benefits from the task sharing. Results are demonstrated through simulation and in reality also.
\end{abstract}

Keywords-Human-Robot Collaboration, industrial robot, simulation, virtual reality

\section{INTRODUCTION}

The paradigm related to Human-Robot Collaboration (HRC) is changing from the separate human from robot (past) to improved human access to robot (present) and close humanrobot interaction (future). One major problem for the introduction of robots especially in unstructured environment is the possibility to rely on dependable sensors. Sensor data are needed for reactive planning, motion/force control, visual servoing, fault diagnosis, and monitoring of safety levels. If the HRC system is planned for unstructured environments with unpredictable movements of persons, HRC should be equipped with a versatile sensor system, including: range, proximity, touch, vision, sound, temperature, and so on. The selection, the arrangement, the number of sensors and their reliability contribute to the measure of dependability of a manipulator for interaction tasks [1].

There are lot of sensors available for HRC systems. Close Human-Robot Collaboration with advanced safety sensors may support speed \& separation monitoring and safety rated monitored stop modes. These might include close proximity sensors, such as pan/tilt/zoom cameras, stereo cameras, depth cameras, projection based-systems [2], and audio/video feedback systems. A better fit for traditional robots (large, high speed, high payload) can be achieved with compliment power \& force limiting functions (PFL robots) [3]. Sensors for distance interaction include pan/tilt/zoom cameras, stereo cameras, projection based-systems, 3D Lidar [4], audio/video feedback systems, certified safety sensors. HRC sensors can also include force/torque sensors or proximity sensors to be used integrated to grippers. Fig. 1 shows an example configuration of the dynamic safety system with sensors [4] together with an example of advanced lidar sensors.

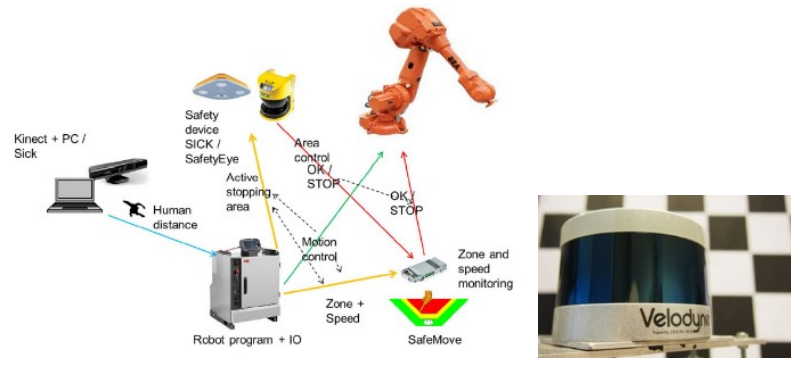

Fig. 1. An example of a dynamic safety system which uses multiple sensors and one potential lidar sensor [4] which is already in use in GIM Ltd robotic solutions [6].

However, the sensors and other devices may vary depending on the complexity of the needed safety system. There are also many standards which have to take into account when choosing sensors, like Omron STI presentation shows in Fig. 2.

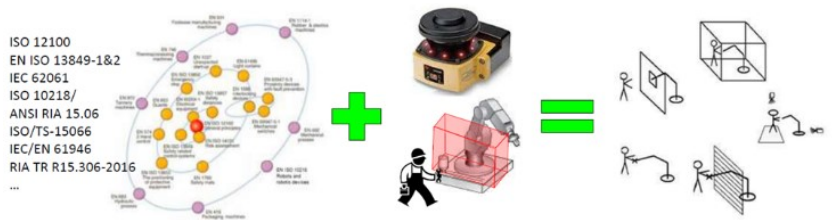

Fig. 2. Human-robot collaboration is changing and there are many standards which are related to it [7]

Fusion of the information coming from multiple sensors may help in providing a coherent and reliable description of the world surrounding the robot. In general, it is required to integrate sensor information based only on approximate models of the environment. Data fusion is particularly important when monitoring contacts, e.g. for selecting impedance parameters or for determining the most dangerous "control points" on the robot to be driven away from a human with higher priority [8]. Unfortunately, there has been little work on achieving the fusion of contact and visual information.

Collaborative robot is defined in standard ISO 10218-2 as follows: Robot designed for direct interaction with a human 
within a defined collaborative workspace i.e. workspace within the safeguarded space where the robot and a human can perform tasks simultaneously during production operation. Basically, the idea is that robot does not hurt a person and the means to protect a person are controlled force and speed, separation monitoring, hand-guiding and safety-rated monitored stop. Fig. 3 shows the means that can be applied in manual or collaborative operation.

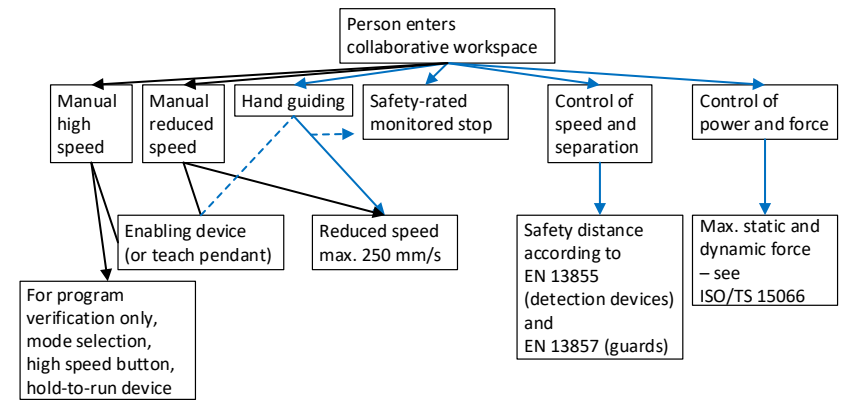

Fig. 3. Collaborative and manual modes applied in collaborative workspace [9].

Different Human-Robot Interaction (HRI) will happen when human collaborate with robot. A new classification strategy has been proposed depending on the level of HRI. According to this approach, most of the possible HRI in industry could be classified into four levels of interaction [10], see in Fig. 4.

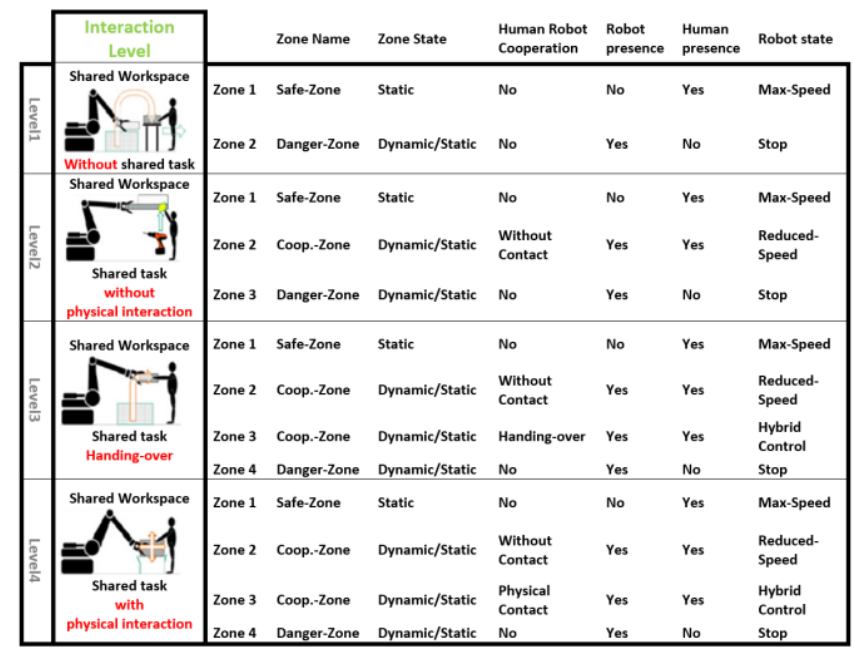

Fig. 4. Four levels of HRI [10].

1) Shared workspace without shared task: The robot and the human acting in a shared fenceless workplace but working on their own task each other.

2) Shared workspace, shared task without physical interaction: The robot and the human have a shared task, but no direct interaction. The robot can only move to a predefined position near human for assisting.

3) Shared workspace, shared task 'handing-over': The shared task consists of a direct handing-over between robot and human, but no physical interaction.

4) Shared workspace, shared task with physical interaction: The robot and the human working in a task which physical interaction is necessary.

\section{LITERATURE REVIEW ON HRC WITH VIRTUAL REALITY}

Nowadays, with the development of industrial 4.0 technologies, more and more network cameras and sensors have been adopted during human-robot collaboration [11]. Researchers also developed various way to use these cameras and sensors. Such as in [12], the author showed a method which using augmented reality (AR) technologies in a mobile platform to control a real robot. A tablet camera offers a real-time video stream to a server through wireless communication, and the tablet feedback 3D graphics with touchscreen interaction to users. It allows user to effectively communicate with robot.

Due to various reasons, such as human safety, space limitation or price, people cannot directly conduct experiment on real robot. Then, more researchers use the cameras and sensors combining with Virtual Reality (VR) technology to conduct experiments in simulation. In [13], the author introduced a simulated hand guiding robot system with a using of force feedback device. In the VR simulation, it allows user to move the robot (which with a screw driver mounted) simply and intuitively on the target place (on the screw) and let the robot out put the correct torque. In [14], the paper introduced a simulated robot controller using Unity built-in kinematics, achieved a realtime controlling of a specific type of ABB robot. The author using Robot Operating System (ROS) as middleware driving the robot in VR world, and feedback to user by HTC Vive. In [15], the paper also introduced a simulated robot controller, but implement a special designed forward and inverse kinematics algorithms in MATLAB. The robot controller can manipulate specific type of KUKA robot in real-time under virtual reality environment. And the author also explained a possibility to connect the VR model with real robot. In [16], the paper introduced a prototype which using proactive and adaptive techniques to avoid possible collision between robot and human. After setting up Microsoft Kinect (as an input for skeletal tracking of the user) and putting on Oculus Rift DK2 (as an output device for stereoscopic visual display, and as an input device for head motion tracking), the user can interact with the prototype in virtual reality environment. In [17], the author using Oculus Rift DK2 as visual display, and a tracking system that tracks the user's head position, pose, and eye-gaze, achieved controlling a real Baxter robot to pick up a part. And a simulated robot moving synchronously.

With the help of VR simulation, researchers really make human-robot collaboration more flexible and intuitive, and more experiments can be conducted to test different new ideas.

\section{COLLABORATION IN VIRTUAL REALITY}

With the widespread of automation and industrial robotics, there are new tasks, which human and an industrial robot could solve together. One of the tasks, which human solves effectively is placing a nut on a screw. This is however challenging in cases, where the screw and the nut is heavy and a worker needs to do this repetitive task all day long. Our solution provides ergonomic solution for this problem, as it provides a high level HRI to relieve human from the tedious work. It is very easy to construct an HRC scenario that the robot picks up the screw and put it at a predefined position, then the human put a nut on screw, rotate the screw. But this is only level 2 in the HRI, we could 
make the robot even more helpful with the HRI is level 4. Then the robot interacts with human directly, helping human put the nut on the screw and rotate the screw. All the human worker need to do is just move a nut near to robot (no matter which direction) and trigger the robot, then let the robot finishing the rest of the task.

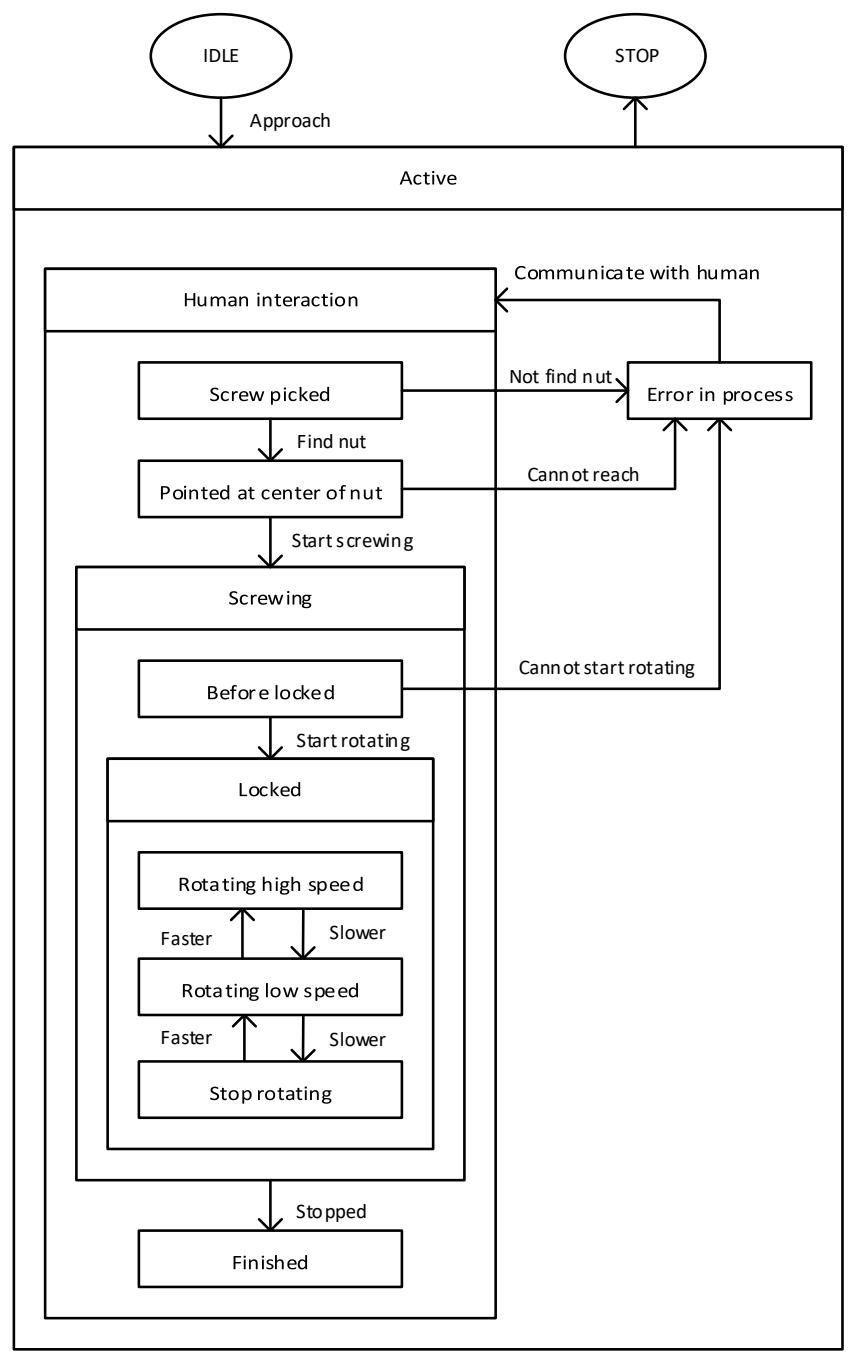

Fig. 5. State flow.

The process of the whole HRC can be described as different processes. Human approach to the robot working area triggering robot from idle state to active. During human-robot interaction, if any error in the process, robot will pop-out error message to a screen and communicate with human. The human interaction process contains:

1) Screw picked: Robot picks up the screw, hold it by gripper. If system cannot find the screw, output an error.

2) Pointed at center of nut: System find out the coordinate of nut, then robot move the screw to nut position with pointing at the center of nut. If robot cannot reach to the target place, output an error.

3) Screwing: Start the physical interaction.
4) Finished: The screwing task finished, back to idle state or stop state.

Human operator can trigger the robot processes by different voice instructions. During the screwing process, there are two stages, before the screw/nut locked and after screw/nut locked. If human moved the nut position beyond a certain tolerance before screw/nut locked, or any other reason causing robot cannot start rotating, an error will be output. After the screw/nut locked, human can change the robot rotating speed from a high speed to stop rotating gradually by voice instructions, also human can stop the whole screwing process and go to next process. See in Fig. 5.

If it is achieved, the difficulty of teaching a robot will dramatically decrease, since there is no specific preprogramming point needed for a robot. And the human worker doesn't need to move nut into specific point to cooperate with robot either. Just enabling the robot, then the robot will collaborate with user automatically. Even no robotic background user can work with robot.

But it is difficult to test the task on real robot directly. Since this task is categorized as level 4 in interaction-levels between human and industrial robot, a lot of safety procedure need to be setup to ensure human worker will not injured before the test, such as monitoring and controlling robot position, speed, torques, and near-field vision system for human hand, body and face [8]. Besides the human safety reason, there are many other advantages of using VR, see the comparison in TABLE 1. In order to test and verify the new idea faster, run a simulation test in Virtual Reality environment will be the best choice.

TABLE 1. COMPARISON BETWEEN VR AND NON VR SCENARIOS

\begin{tabular}{|c|l|l|}
\hline $\begin{array}{c}\text { Locating target } \\
\text { position }\end{array}$ & $\begin{array}{l}\text { Using VR } \\
\text { Simulation software } \\
\text { acquire nut position in }\end{array}$ & $\begin{array}{l}\text { Not using VR } \\
\text { reacial 3D position } \\
\text { detection device must be } \\
\text { installed, and feedback } \\
\text { the coordinate to robot } \\
\text { system }\end{array}$ \\
\hline $\begin{array}{c}\text { Recognizing } \\
\text { target }\end{array}$ & $\begin{array}{l}\text { No special recognition } \\
\text { system needed }\end{array}$ & $\begin{array}{l}\text { System need to setup a } \\
\text { digital database in } \\
\text { advance for recognizing } \\
\text { nut, screw, ball and can }\end{array}$ \\
\hline Human safety & $\begin{array}{l}\text { No special protection } \\
\text { for human }\end{array}$ & $\begin{array}{l}\text { System should trace } \\
\text { human skeleton, and } \\
\text { feedback the 3D } \\
\text { position data to robot } \\
\text { system to avoid hurting } \\
\text { human }\end{array}$ \\
\hline Testing area & $\begin{array}{l}\text { No special testing area } \\
\text { needed }\end{array}$ & $\begin{array}{l}\text { A special testing area } \\
\text { with barrier is needed }\end{array}$ \\
\hline Test calibration & $\begin{array}{l}\text { One button to restore } \\
\text { system starting point }\end{array}$ & $\begin{array}{l}\text { Careful measurement is } \\
\text { needed to keep } \\
\text { experiment in the same } \\
\text { starting point }\end{array}$ \\
\hline
\end{tabular}

\section{EXPERIMENTING WITH COLLABORATION}

According to the proposal, we designed the experiment. The experiment is running under a simulation environment which the simulation software is Visual Components. Visual Components is running in Windows platform which is much user friendly and avoid a lot of coding process comparing with Linux platform. In its own library, Visual Components already embedded abundant robot models, such as ABB, KUKA, FANUC, NACHI, etc. And 
the software offered a Python API to user, so we have the possibility to modify almost every feature of the software.

In Fig. 6, a NACHI MZ07 robot is putted on a work platform, with a simple gripper mounted on. Since the goal is just put a nut on screw, where to achieve the goal is unknown before the system is started. Human worker can move in the nut from any direction, we define the robot motion not based from any specific point. So, in the initial state, a nut with a random coordinate on a human worker's hand. And a screw with a random coordinate in a box. Inside the box, there are also a can and a ball. A camera mounted on a pillar to capture and recognize different objects with their coordinates. After the human worker triggered the system by press the switch under the foot (simulation started), the background Python script can access to the objects' coordinates and generate a series of predefined robot point-to-point (PTP) or linear (LIN) motion statements.

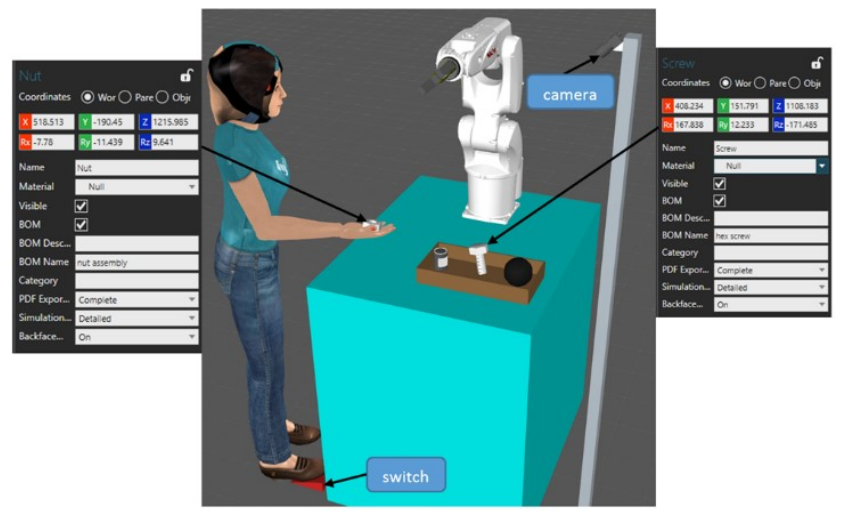

Fig. 6. Pick the screw, put at the center of nut.

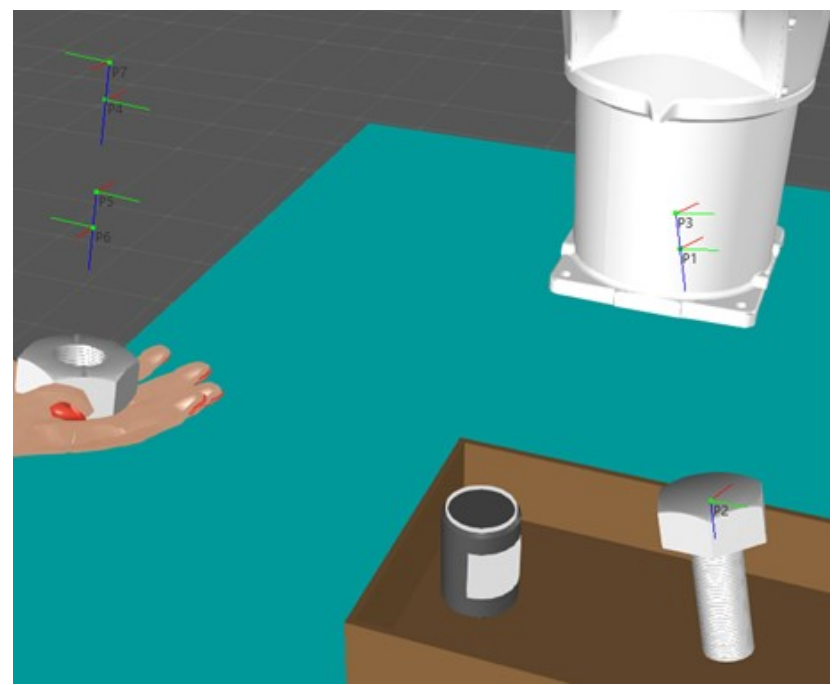

Fig. 7. The robot Tool Centre Point (TCP) represented by each statement respectively

In Fig 7, we can see the corresponding TCP positions represented by each robot motion statement. The goal which putting a nut on screw should also contain a basic method to teach robot finishing the task. The robot motion statement is generated by this method. In this method, after the system acquired the information of nut and screw position (can be input from camera system or simulation initial state), the TCP position in each motion statement will be re-calculated and updated. With this method, our goal can be achieved, and a level 4 interaction could be conducted between human and robot through the VR environment.

In Fig. 8, we can see a sample execution of the previously described interaction. The sequence of the movement are determined by the state flow presented in Fig. 5, but the actual position coordinates are calculated real-time in the simulation environment. The data necessary to decide about positions are gathered from the virtual reality and the reality (physical robot). The connection between these two are based on a networked solution previously developed in-house, which can control the physical robot from an external device on high frequency (83 $\mathrm{Hz})[18]$

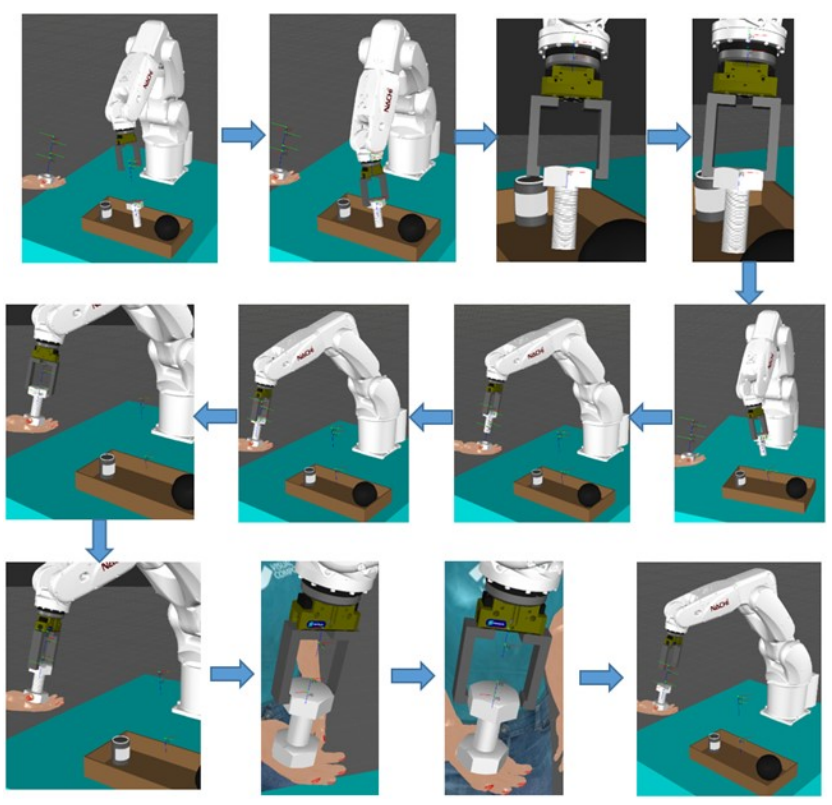

Fig. 8. Movement of the robot

The human can follow the state of the interaction through a Graphical User Interface (GUI). This GUI describes the current interaction, information on the next steps and if there any errors, that needs attention from the human operator. Not only the GUI, but other sensors are also used in order to detect the human's intentions. Two depth cameras are installed to detect human and nut in real-time. Microsoft Kinects are chosen as these have the necessary speed and are well known and used in humanmachine interaction scenarios. One is used to detect human skeleton pose and another one is focused on human hand and nut for higher accuracy. After the nut and human positions are detected, the information is updated in the simulation software and on the physical robot also.

A video recording of the interaction can be viewed at: http:/vizlab.uit.no/iecon2018/ 


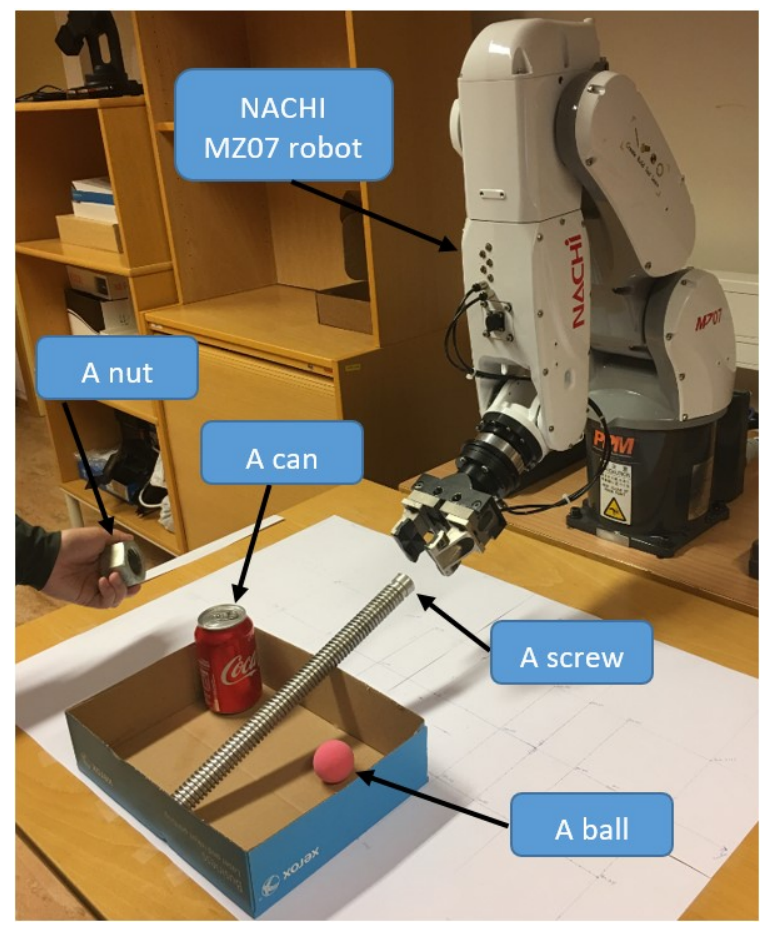

Fig. 9. The proposed HRC in real world.

In Fig. 9, we can see the proposed HRC in real world. A human holding a nut, waiting the robot to pick the screw from the box and working together. With the successful of preliminary idea test in VR environment, we can conduct this real test in next stage.

\section{CONCLUSION}

Human-Robot Collaboration is not any more limited to sharing the space with an industrial robot. In our experiments we show how given simple task could be shared between the human operator and the industrial robot. The experiment shows the feasibility of the approach and the Virtual Reality system helps the operator to achieve the necessary comfort functions, which is needed for a level 4 Human-Robot Collaboration.

\section{FUTURE WORK}

To achieve this robot movement, future work should be focused on four main tasks:

- A camera/sensor system which should not only can recognize the nut and screw but also can calculate their coordinates in 3D space.

- A server can calculate the robot joint angle based from the $3 \mathrm{D}$ coordinates.

- The robot can move to the desired position by receiving the joint angle value from server.

- Human safety procedures.
At last, a synchronization between the real world and the virtual world should be setup.

\section{REFERENCES}

[1] R. Alami, A. Albu-Schaeffer, A. Bicchi, R. Bischoff, R. Chatila, A. De Luca, et al. "Safe and dependable physical human-robot interaction in anthropic domains: State of the art and challenges," in IEEE International Conference on Intelligent Robots and Systems. 2006.

[2] C. Vogel, M. Poggendorf, C. Walter and N. Elkmann. "Towards safe physical human-robot collaboration: A projection-based safety system," in IEEE International Conference on Intelligent Robots and Systems. 2011.

[3] N. Ando, P. T. Szemes, P. Korondi and H. Hashimoto, "Friction compensation for 6DOF Cartesian coordinate haptic interface," IEEE/RSJ International Conference on Intelligent Robots and Systems, Lausanne, Switzerland, 2002, pp. 2893-2898 vol.3.

[4] 3D Lidar http://www.velodynelidar.com/.

[5] T. Salmi, O. Väätäinen, T. Malm, J. Montonen and I. Marstio, "Meeting New Challenges and Possibilities with Modern Robot Safety Technologies," in Enabling Manufacturing Competitiveness and Economic Sustainability. 2014. pp. 183-188.

[6] GIM Ltd robotic solutions http://gimltd.fi/services.html.

[7] T. Knauer, "Safety Sensor Technologies Enabling Closer Human-Robot Collaboration," in AUTOMATE 2017, 2017.

[8] P. Korondi, D. Young and H. Hashimoto, "Sliding mode based disturbance observer for motion control," Proceedings of the 37th IEEE Conference on Decision and Control (Cat. No.98CH36171), Tampa, FL, USA, 1998, pp. 1926-1927 vol.2.

[9] T. Malm, T. Salmi, I. Marstio and J. Montonen, "Safe collaboration of operators and industrial robots," in Automaatiopäivät22 2017 Seminar, 2017.

[10] M. Bdiwi, M. Pfeifer, and A. Sterzing, "A new strategy for ensuring human safety during various levels of interaction with industrial robots," CIRP Annals, 2017, vol. 66(1), pp. 453-456.

[11] Dániel, B., Korondi, P., Sziebig, G. and Thomessen, T., "Evaluation of flexible graphical user interface for intuitive human robot interactions," Acta Polytechnica Hungarica, 11(1), 2014, pp.135-151.

[12] J.A. Frank, M. Moorhead, and V. Kapila. "Realizing mixed-reality environments with tablets for intuitive human-robot collaboration for object manipulation tasks," in 2016 25th IEEE International Symposium on Robot and Human Interactive Communication (RO-MAN). 2016.

[13] U. Dombrowski, T. Stefanak, and J. Perret, "Interactive Simulation of Human-robot Collaboration Using a Force Feedback Device," Procedia Manufacturing, 2017, vol. 11, pp. 124-131.

[14] A. de Giorgio, M. Romero, M. Onori, and L. Wang, "Human-machine Collaboration in Virtual Reality for Adaptive Production Engineering," Procedia Manufacturing, 2017, vol. 11, pp. 1279-1287.

[15] L. Gammieri, M. Schumann, L. Pelliccia, G. Di Gironimo, and P. Klimant, "Coupling of a Redundant Manipulator with a Virtual Reality Environment to Enhance Human-robot Cooperation," Procedia CIRP, 2017, vol. 62, pp. 618-623.

[16] E. Matsas, G.-C. Vosniakos, and D. Batras, "Prototyping proactive and adaptive techniques for human-robot collaboration in manufacturing using virtual reality," Robotics and Computer-Integrated Manufacturing, 2018, vol. 50, pp. 168-180.

[17] M.M. Moniri, F.A.E. Valcarcel, D. Merkel, and D. Sonntag, "Human Gaze and Focus-of-Attention in Dual Reality Human-Robot Collaboration," in 2016 12th International Conference on Intelligent Environments (IE), 2016.

[18] P. Korondi and J. Gyeviki, "Robust Position Control for a Pneumatic Cylinder," 2006 12th International Power Electronics and Motion Control Conference, Portoroz, 2006, pp. 513-518. 